\title{
Tunable Band Gap of Boron Nitride Interfaces under Uniaxial Pressure
}

\author{
Elizane E. Moraes, Taíse M. Manhabosco, Alan B. de Oliveira, and Ronaldo J. C. Batista \\ Departamento de Física, Universidade Federal de Ouro Preto, \\ Ouro Preto, MG, 35400-000, Brazil
}

(Dated: August 25, 2018)

\begin{abstract}
In this work we show, by means of a density functional theory formalism, that the simple physical contact between hydrogen terminated boron nitride surfaces gives rise to a metallic interface with free carries of opposite sign at each surface. A band gap can be induced by applying uniaxial pressure. The size of the band gap changes continuously from zero up to $4.4 \mathrm{eV}$ with increasing pressure, which is understood in terms of the interaction between surface states. Due to the high thermal conductivity of cubic boron nitride and the coupling between band gap and applied pressure, such tunable band gap interfaces may be used in high stable electronic and electromechanical devices. In addition, the spacial separation of charge carries at the interface may lead to photovoltaic applications.
\end{abstract}


Diamond is commonly used as an abrasive material in technological and industrial applications due to its hardness. Other intrinsic properties of diamond have increased its range of applications from biotechnology to electronics ${ }^{1-4}$. In electronics, in particular, its large electronic band gap and exceptionally high thermal conductivity led to the production of highly stable solid state transistors (FETs) based on hydrogen terminated diamond $\underline{-3}-\underline{\underline{5}}$. Due to the similarity between the isoelectronic $\mathrm{C}-\mathrm{C}$ and $\mathrm{B}-\mathrm{N}$ bonds, boron and nitrogen form a material analogous to the carbon diamond, namely, cubic boron nitride (cBN). cBN presents hardness, band gap and thermal conductivity very similar to those of carbon diamond. In addition, it is also possible to produce hydrogen terminated $\mathrm{cBN} \underline{6}$ surfaces. Nevertheless, the $\mathrm{C}-\mathrm{H}$ is not isoeletronic to $\mathrm{B}-\mathrm{H}$ and $\mathrm{N}-\mathrm{H}$ bonds, thus hydrogen terminated diamond and hydrogen terminated $\mathrm{cBN}$ must present distinct electronic and electrochemical properties. For example, the band gap of thin hydrogen terminated cBN films depends on the number of BN layers due to the differences between $\mathrm{B}-\mathrm{H}$ and $\mathrm{N}-\mathrm{H}$ terminated surfaces ${ }^{7}$. On the other hand, the band gap of thin diamond films is constant.

In this work, we apply a density functional formalism to investigate interfaces formed by the physical contact between hydrogen terminated boron nitride surfaces. We found that those interfaces present a band gap that can be continuously controlled by applied uniaxial pressure, which is explained in terms of the interaction between surface states due to the B-H and N-H terminations. The gap of the system can be modified from zero up to roughly $4.4 \mathrm{eV}$ (a value of band gap close to that calculated for $\mathrm{cBN}$ within density functional theory approach). At null values of pressure the interface presents a metallic behavior with electrons at N-H terminated surface and holes a B-H terminated surface. The semiconductor, metallic and insulating behavior of such interfaces may allow their use in electronics as high stable devices. The coupling between applied pressure and the band gap, on the other hand, may permit the use of such interfaces in electromechanical devices. Besides, since the free carries are spatially separated in those interfaces and the $\mathrm{cBN}$ is transparent for visible light, they could be also used in photovoltaic applications.

The applied first-principles methodology is based on the Density Functional Theory (DFT) as implemented in the SIESTA program $\stackrel{8}{*}$. We used the Generalized Gradient Approximation (GGA) as parametrized in the Perdew-Burke-Ernzerhof scheme $(\mathrm{PBE})^{\underline{9}}$ for the exchange-correlation functional. The ionic core potentials were represented by normconserving scalar relativistic Troullier-Martins ${ }^{10}$ pseudopotentials in Kleinman-Bylander 


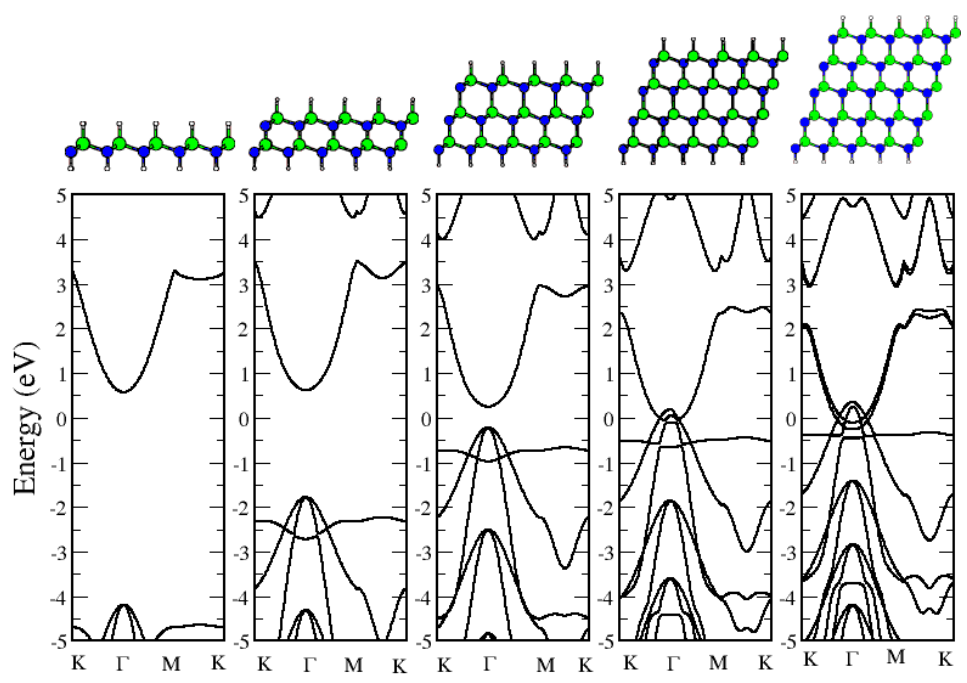

FIG. 1: Bands structure of thin boron nitride nanofilms. From left to right the number of layers increases from one up to five. As the number of layers decreases the overlap between orbitals of atoms at each surface increases giving rise to band gaps.

nonlocal form $\underline{11}$. The fineness of the real-space grid integration was defined by a minimal energy cutoff of $150 \mathrm{Ry} \underline{\underline{12}}$. The geometries were fully optimized using the conjugate gradient algorithm $\frac{13}{\underline{3}}$ until all the force components were smaller than $0.02 \mathrm{eV} / \AA$. The Kohn-Sham (KS) eigenfunctions were expanded as linear combination of pseudo atomic orbitals of finite range consisting of double-zeta radial functions per angular momentum plus polarization orbitals (DZP).

As a first step to understand the electronic properties of $\mathrm{cBN}$ interfaces we investigated few-layered hydrogenated (111) BN films. The top panels of Fig. 1 show the geometries of the few-layered films whose number of layers changes from one up to five while the bottom panels show the respective electronic structure. It is clear that there is a dependence of the band gap with the number of layers, which can be understood in terms of differences between $\mathrm{B}-\mathrm{H}$ and $\mathrm{N}-\mathrm{H} s p^{3}$ terminations.

The C-C $s p^{3}$ bonds in diamond share a pair of electrons (satisfying then the octet rule) which leads to a wide band gap between valence and conducting states. Since boron and nitrogen are the carbon left and right neighbors in the periodic table of elements, respectively, the $\mathrm{B}-\mathrm{N}$ bond is isoelectronic to $\mathrm{C}-\mathrm{C}$ bond. Therefore, the $\mathrm{B}-\mathrm{N} s p^{3}$ bonds in $\mathrm{cBN}$ also share a pair of electrons where the excess of electrons of nitrogen orbitals are precisely offset by 
the lack of electrons in boron orbitals. As a result, cBN presents a band gap as wide as that of carbon diamond. In the $\mathrm{N}-\mathrm{H}$ and $\mathrm{B}-\mathrm{H}$ bonds on hydrogen terminated $\mathrm{cBN}$, on the other hand, the $\mathrm{H}$ atoms do not compensate the excess or lack of electrons on $\mathrm{N}$ and $\mathrm{B}$ orbitals in cBN surfaces, which results in surface states within the large band gap of cBN. In a sufficiently thick film the interaction between those surfaces is negligible. This is the case of films with four layers or more, in which there is no overlap between N-H and B-H orbitals (indeed, the distance between $\mathrm{N}$ and $\mathrm{B}$ atoms at both ends in the 4-layer BN film is $7.12 \AA$, that is greater than two times the cutoff radius of $\mathrm{N}$ and $\mathrm{B}$ orbitals, $2.5 \AA$ ). In those cases, the edge of the occupied surface state localized at the B-H end is above the edge of the unoccupied surface state localized at the N-H end (the fourth and fifth panels of Fig. 1 show a superposition of non-interacting surface states). Thus, the N-H terminated surface can transfer charge to the B-H terminated surface if a physical contact is provided. In a very recent work ${ }^{14}$, we have investigated by means of theory and experiment a similar case in which the physical contact between semiconducting carbon nanotubes (SCNT) and diamond surfaces - which are large gap insulators - allows a metallic response. In that case, the top of valence band of diamond surface was above the bottom of the conduction band of SCNT.

A physical contact between N-H and B-H terminated surface occurs in films with less than 3 layers since there is an overlap between orbitals of $\mathrm{B}$ and $\mathrm{N}$ atoms at different surfaces. In a single layer film (left panel of Fig. 1) the overlap between $\mathrm{N}$ and B orbitals is maximum, then the excess or lack of electrons at N-H and B-H surfaces is canceled out. As a result the one layer $\mathrm{cBN}$ film presents a band gap very similar to that of $\mathrm{cBN}$ bulk. The overlap between orbitals of the atoms at different surfaces decreases with film thickness which leads to values of band gaps shown in the second and third panels of Fig. 1.

The results discussed above strongly suggest that the overlap between orbitals of the atoms of B-H and N-H cBN surface determine the band gap since it affects charge distribution that gives rise to the surface states. The overlap between the orbitals of atoms at different surfaces can be continuously controlled by the physical contact between surfaces as shown in the left panel of Fig. 2. We have simulated the physical contact between B-H and N-H terminated cBN surfaces by performing calculations on 4-layer cBN nanofilms in which he distance between successive periodical images allows the interaction between B-H and N-H terminated surfaces. The right panel of Fig. 2 shows the band structure of an 


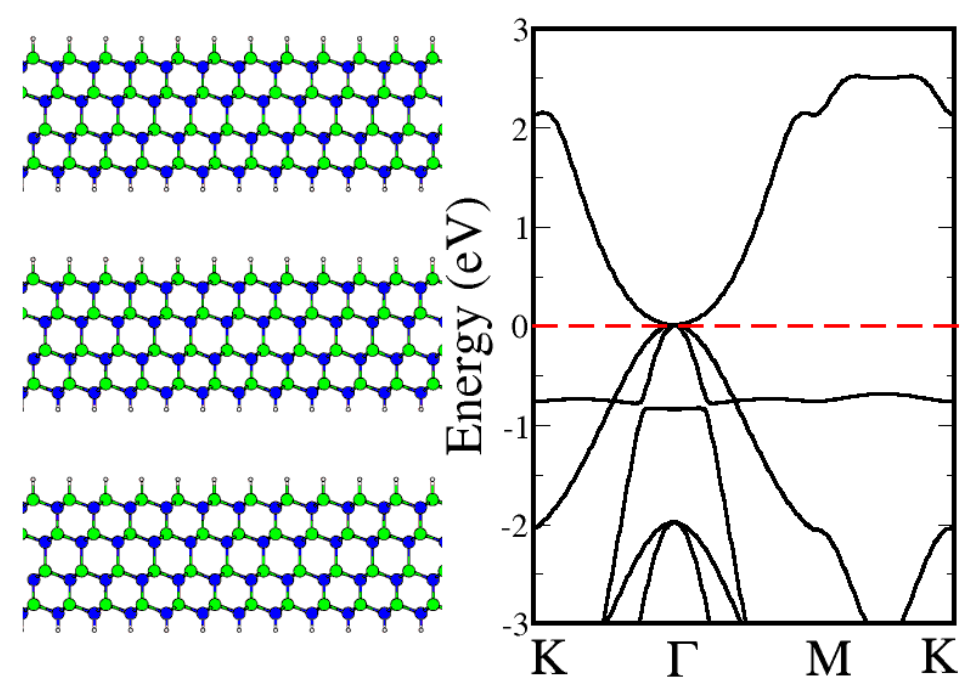

FIG. 2: Left panel: interfaces formed by the physical contact between N-H and B-H terminated cBN four-layered films, only the first neighbors periodical images are shown. Right panel: bands structure of the interfaces shown in the left panel.

interface formed by the simple deposition of a $\mathrm{H}$ terminated cBN surface onto its counterpart, at null values of uniaxial pressure. It is possible to see that the band structure of such an interface differs from the band structure of an isolated four layer film (fourth panel of Fig. 1). Such a difference is due to a charge transfer between the B-H and N-H surfaces that changes the position of surface states. In spite of that, the net charge transferred is not enough to open a band gap in the interface. Then, the N-H/B-H interface is metallic with free charge carries of opposite sign at each surface. The positive charge will, therefore, attract the negative charge at adjacent surface, thereby producing a bidimensional electron gas. The combination of bidimensional electron and hole gases, very close to each other, could lead to interesting transport properties.

The applied uniaxial pressure decreases the distance between B-H and N-H surfaces at the interface that increases the overlap between orbitals of atoms at the surfaces. The left panel of Fig. 3 shows the band of gap of the interface as a function of the overlap volume between $s \mathrm{H}$ orbitals $\frac{15}{15}$. It is possible to see a precise linear dependence of the band gap with the overlap, which corroborate the importance of overlap between orbitals of atoms at surface for the band gap of few-layered films. The value of pressure that corresponds to the greatest value of overlap in Fig. 2 is about $70 \mathrm{Kbar}$, which can be achieved experimentally. 

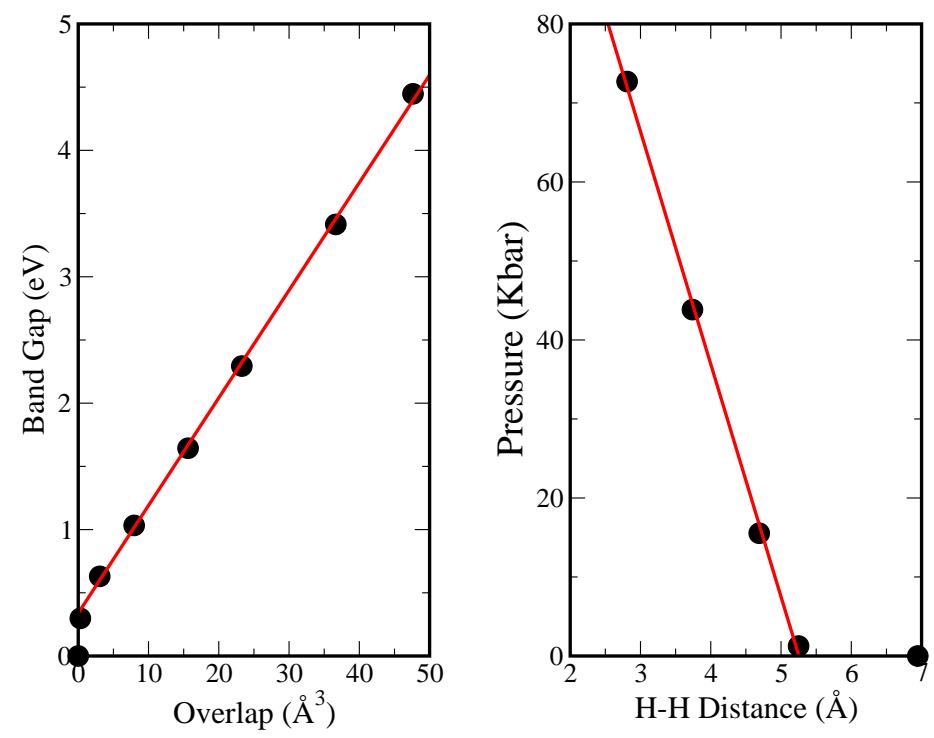

FIG. 3: Left panel: band gap as a function of the overlap volume between hydrogen $s$ orbitals of $\mathrm{H}$ atoms at the interface formed by the physical contact between $\mathrm{B}-\mathrm{H}$ and $\mathrm{N}-\mathrm{H}$ terminated cubic boron nitride, see left panel of Fig. 2. Right panel: uniaxial pressure as a function the distance between hydrogen atoms at B-H terminated surface and $\mathrm{N}-\mathrm{H}$ terminated surface.

Thus, any value of band gap ranging from zero up to $4.4 \mathrm{eV}$ in the interface formed by the physical contact between B-H and N-H cBN surfaces can be tuned through the uniaxial applied pressure.

In summary, we report first-principles calculations on a tunable band gap interface composed of a high stable material, the cubic boron nitride. Such result is very interesting in a applied point of view since: (i) high stable tunable semiconductors are highly desired for electronic applications; (ii) the continuous dependence of the band gap with the applied pressure may be useful in very accurate electromechanical devices; (iii) the spatial separation of the charge carries of opposite sign at the interface combined with the transparency of cBN bulk for visible light may allow the production of photovoltaic devices. The mechanism whit leads to the dependence of the band gap with applied pressure is understood in terms of the interaction between surface states. 


\section{Acknowledgments}

The authors acknowledge financial support from CNPq, FAPEMIG, Rede Nacional de Pesquisa em Nanotubos de Carbono, and INCT-Nano-Carbono.

1 Manhabosco T M and Müller I L 2009 Appl. Surf. Sci. 255, 4082-4086.

2 Manhabosco T M and Müller I L 2009 Tribol. Lett. 33, 193-197.

3 Denisenko A and Kohn E 2005 Diamond Relat. Mater. 14, 491-498.

4 Moran D A J, Fox O J L, McLelland H, Russell S, and May P W 2011 IEEE Electron Device Lett. 32, 599-601.

5 Taniuchi H, Umezawa H, Arima T, Tachiki M, and Kawarada H 2001 IEEE Electron Device Lett. 22, 390-392.

6 Reinke P, Oelhafen P, Feldermann H, Ronning C and Hofsäss 2000 J. Appl. Phys. 88, 5597-5604.

7 Zhang Z and Guo W 2012 Nano Lett. 12, 3650-3655

8 Soler J M, Artacho E, Gale J D, Garcia A, Junquera J, Ordejón P and Sánchez-Portal D 2002 J. Phys.: Condens. Matter 14, 2745-2779.

9 Perdew J P, Burke K and Ernzerhof M 1996 Phys. Rev. Lett. 77, 3865-3868.

10 Troullier N, Martins J L 1991 Phys. Rev. B 43, 1993-2006.

11 Kleinman L and Bylander D M 1982 Phys. Rev. Lett. 48, 1425-1428

12 Moreno J and Soler J M 1992 Phys. Rev. B 45, 13891-13898.

13 Payne M C, Teter M P, Allan D C, Arias T A and Joannopoulos 1992 Rev. Mod. Phys. 64, 1045-1097.

14 Barboza A M, Carara S S, Batista R J C, Chacham H and Neves B R A Small 8, 220 (2012)

15 The overlap volume between two identical spheres is given by: $V=\frac{\pi}{12}(4 R+d)(2 R-d)^{2}$, where $R$ is the sphere radius and $d$ is the distance between centers. 